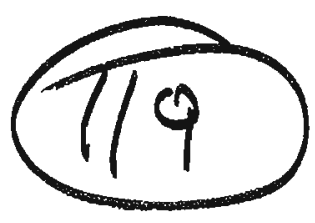

(c) Springer-Verlag 1998

\title{
Block LU factorizations of M-matrices
}

\author{
J.J. McDonald ${ }^{1, \star}$, H. Schneider ${ }^{2, \star \star}$ \\ 1 Department of Mathematics and Statistics, University of Regina, Regina, Saskatchewan, \\ Canada, S4S OA2 \\ 2 Department of Mathematics, University of Wisconsin, Madison, WI 53706, USA
}

Received November 21, 1994 / Revised version received August 4, 1997

Summary. It is well known that any nonsingular M-matrix admits an LU factorization into M-matrices (with $L$ and $U$ lower and upper triangular respectively) and any singular $\mathbf{M}$-matrix is permutation similar to an $\mathbf{M}$-matrix which admits an LU factorization into M-matrices. Varga and Cai establish necessary and sufficient conditions for a singular M-matrix (without permutation) to allow an $L U$ factorization with $L$ nonsingular. We generalize these results in two directions. First, we find necessary and sufficient conditions for the existence of an LU factorization of a singular M-matrix where $L$ and $U$ are both permitted to be singular. Second, we establish the minimal block structure that a block LU factorization of a singular M-matrix can have when $L$ and $U$ are $\mathbf{M}$-matrices.

Mathematics Subject Classification (1991): 65F05

\section{Introduction}

It was shown by Fiedler-Ptak, [3], that any nonsingular M-matrix $A$ admits an LU factorization, $A=L U$, where $L$ is a nonsingular lower triangular Mmatrix, and $U$ is a nonsingular upper triangular M-matrix. Kuo, [8], proved that any (singular) irreducible M-matrix $A$ admits an LU factorization, $A=$ $L U$, where $L$ is a nonsingular lower triangular $\mathbf{M}$-matrix, and $U$ is a upper triangular M-matrix, and she gave an example to show that not every singular M-matrix admits an $L U$ factorization of this type. In [13], Varga and Cai

\footnotetext{
* Work supported by an NSERC Research Grant

** Work supported by NSF Grant DMS-9123318 and DMS-9424346

Correspondence to: $\mathrm{H}$. Schneider
} 
establish necessary and sufficient conditions in terms of the directed graph $\mathcal{G}(A)$ of $A$ for a singular M-matrix to allow a $L U$ factorization into $\mathrm{M}$ matrices with $L$ nonsingular.

In this paper we consider the case where the conditions outlined in [13] may not be satisfied. We generalize these results in two directions. First, we find necessary and sufficient conditions for the existence of an LU factorization of a singular M-matrix where both $L$ and $U$ are permitted to be singular. Second, we wish to factor an $\mathrm{M}$-matrix $A$ into $A=L U$, where $L$ and $U$ are $\mathrm{M}$-matrices which are as close to lower and upper triangular as possible. Our goal is to minimize the number of nonzeros above the diagonal of $L$ and below the diagonal of $U$ in a factorization $A=L U$ and/or to optimize on their placement. Our approach to this problem is to minimize the appropriate access relationships in the digraphs $\mathcal{G}(L)$ and $\mathcal{G}(U)$ of $L$ and $U$ respectively. In order to establish the minimal block structure that a block LU factorization of a singular M-matrix can have we need to give careful definitions of what is meant by the block lower (upper) triangular self-partition of a matrix. These partitions are minimal in the set of partitions that lead to a block lower (upper) triangular matrix and they are solely determined by the zero/non-zero pattern of the matrix and thus do not depend on some assumed prior partitioning of the matrix. We use the term block factorization to indicate that we are interested in the block structure (without permutation) of the factors involved.

We now describe our paper in more detail. Our definitions are contained in Sect. 2.

In Sect. 3 we examine both LU and block LU factorizations of Mmatrices where $L$ and $U$ are permitted to be singular. Thus, in Example 3.1 we provide an M-matrix $A$ which has an LU factorization into Mmatrices only when $L$ and $U$ are both singular. In Theorem 3.5 we identify the minimum access relationships which must be present in $\mathcal{G}(L)$ and $\mathcal{G}(U)$ and thus also identify the minimum sizes of the diagonal blocks of $L$ and $U$ in a block $L U$ factorization. In Theorem 3.7 we show that these minimum block structures can be achieved. Returning to (elementwise) LU factorizations in Theorem 3.9, we there characterize the M-matrices $A$ which admit an LU factorization into (possibly singular) $\mathrm{M}$-matrices. We give two strategies, one for finding a desirable block $L U$ factorization, and the other for choosing a permutation matrix $P$ so that $P A P^{\mathrm{T}}$ has an $\mathrm{LU}$ factorization.

In Sect. 4 we consider the case where $L$ is a nonsingular M-matrix and $U$ is an M-matrix. Using definitions and a result from McDonald [9], we actually examine a slightly larger class - factorizations of an M-matrix $A$ for which $L$ is nonsingular and inverse nonnegative, $L^{-1}$ is class nonsingular for $A$, and $U$ is an M-matrix. In Theorem 4.1 we show that for such a factorization, certain access relationships must be present in $\mathcal{G}(U)$ and thus 
put a lower limit on the number of nonzeros below the diagonal of $U$. In Theorem 4.2 we show that we can attain these minimum access relationships using a factorization for which $L$ is a lower triangular nonsingular $\mathrm{M}$-matrix.

In Sect. 5 we examine factorizations of $A$ into $L B U$ where $L$ is a nonsingular lower triangular M-matrix, $U$ is a nonsingular upper triangular $\mathrm{M}$-matrix, and $B$ is a block diagonal M-matrix. Since $L$ and $U$ are nonsingular lower and upper triangular $M$-matrices, standard methods could be used to solve the parts of the system in which they are involved. Since $B$ is generally a sparse matrix, it may be possible to use specialized techniques in this area to solve the part of the system involving $B$.

We remark that analogous results hold for UL-factorizations and that the techniques used in this paper can also be used to identify the minimum structures of $U$ and $L$ in this instance.

Our results are of a graph theoretical nature, but we express the hope that our identification of possible block LU and LBU factorizations of singular M-matrices will extend applications and numerical implementations of block LU and LBU factorizations such as those discussed in [6, Chap. 12].

\section{Definitions}

We begin with some standard definitions. Let $n$ be a fixed positive integer. We write $\langle n\rangle=\{1, \ldots, n\}$.

Throughout Sect. 2, $X=\left[x_{i j}\right]$ will denote a matrix in $\mathbb{R}^{n n}$.

We say $X$ is:

positive $(X \gg 0)$ if $x_{i j}>0$, for all $i, j \in\langle n\rangle$;

semipositive $(X>0)$ if $x_{i j} \geq 0$, for all $i, j \in\langle n\rangle$ and $X \neq 0$; and nonnegative $(X \geq 0)$ if $x_{i j} \geq 0$, for all $i, j \in\langle n\rangle$.

We say $X$ is a Z-matrix if $X=\alpha I-P$ for some $\alpha \in \mathbb{R}$ with $P$ nonnegative. If in addition, $\alpha$ is greater than or equal to the spectral radius of $P$, then we say $X$ is an $M$-matrix. We denote the class of $n \times n \mathbf{M}$-matrices by $\mathcal{M}$.

For any $J \subseteq\langle n\rangle$, we let

$$
\begin{gathered}
\max (J)=\max \{j \in J\}, \\
\min (J)=\min \{j \in J\}, \\
J^{+}=\{l \in\langle n\rangle \mid l>\max (J)\}, \\
J^{-}=\{l \in\langle n\rangle \mid l<\min (J)\}, \\
J^{\prime}=\{l \in\langle n\rangle \mid l \notin J\} . \\
|J|=\text { number of elements in } J .
\end{gathered}
$$


For any $J, K \subseteq\langle n\rangle$, we write $X_{J K}$ to represent the submatrix of $X$ whose rows are indexed by the elements of $J$ and whose columns are indexed by the elements of $K$, where the elements of $J$ and $K$ are arranged in ascending order.

We call the pair $\Gamma=(V, E)$ a directed graph, where $V$ is a finite set, and $E \subseteq V \times V$. A path from $j$ to $k$ in $\Gamma$ is a sequence of vertices $j=$ $r_{1}, r_{2}, \ldots, r_{t}=k$, with $\left(r_{i}, r_{i+1}\right) \in E$, for $i=1, \ldots, t-1$. A path for which the vertices are pairwise distinct is called a simple path. The empty path is considered to be a simple path linking every vertex to itself. If there is a path from $j$ to $k$, we say that $j$ has access to $k$. If $j$ has access to $k$ and $k$ has access to $j$, we say $j$ and $k$ communicate. The communication relation is an equivalence relation, and hence we may partition $\mathrm{V}$ into equivalence classes, which we will refer to as the classes of $\Gamma$. We define the closure $\Gamma$ by $\bar{\Gamma}=(V, F)$, where $V=\langle n\rangle$ and $F=\{(i, j) \mid i$ has access to $j$ in $\Gamma\}$.

We define the (directed) graph of $X$ by $\mathcal{G}(X)=(V, E)$, where $V=\langle n\rangle$ and $E=\left\{(i, j) \mid x_{i j} \neq 0\right\}$. It is well known that the classes of $\mathcal{G}(X)$ correspond to the irreducible components of $X$. For any class $J$ of $G(X)$ we say that $J$ is a singular class of $X$ if $X_{J J}$ is singular, and we say that $J$ is a nonsingular class of $X$ if $X_{J J}$ is nonsingular.

We commonly view a matrix $X$ as a block matrix

$$
X=\left[\begin{array}{ccc}
X_{11} & \ldots & X_{1 p} \\
\vdots & \ddots & \vdots \\
X_{q 1} & \ldots & X_{q p}
\end{array}\right]
$$

where $X_{i j}$ is $m_{i} \times n_{j}$ with $m_{1}+m_{2}+\ldots+m_{q}=n=n_{1}+n_{2}+\ldots+n_{p}$. In this paper we will require that the diagonal blocks be square, viz. $p=q$ and $m_{1}=n_{1}, m_{2}=n_{2}, \ldots, m_{q}=n_{q}$. Rather than using $m_{1}, m_{2}, \ldots, m_{q}$ to describe our block structure, we will look at the sets $v_{i}=\left\{m_{i-1}+\right.$ $\left.1, \ldots m_{i}\right\}$. More formally, we will say the sequence $\Upsilon=\left(v_{1}, v_{2}, \ldots, v_{r}\right)$ is an (ordered) partition of $\langle n\rangle$, if $\cup_{i=1}^{r} v_{i}=\langle n\rangle$, and $v_{i} \cap v_{j}=\emptyset$, for all $i \neq j$. We say $r$ is an order preserving partition of $\langle n\rangle$ if $\Upsilon$ is a partition such that if $i<j$, then $i \in v_{k_{i}}$ and $j \in v_{k_{j}}$ with $k_{i} \leq k_{j}$. We will say $X$ is block lower triangular with respect to the order preserving partition $\Upsilon=\left(v_{1}, \ldots, v_{r}\right)$ if $X_{v_{i} v_{i}^{+}}=0$, for every $i \in\langle r\rangle$. Clearly $X$ may be block lower triangular with respect to several different partitions. We call $\Psi=\left(\psi_{1}, \psi_{2}, \ldots, \psi_{p}\right)$ a refinement of an order preserving partition $\Upsilon$, if $\Psi$ is also an order preserving partition of $\langle n\rangle$ and if for every $i \in\langle p\rangle$ there exists $j \in\langle r\rangle$ such that $\psi_{i} \subseteq v_{j}$. A refinement is said to be proper if $\Psi \neq \Upsilon$. The refinement relation on the set of order preserving partitions of $\langle n\rangle$ defines a lattice such that greatest lower bound of two partitions has as its elements intersections of the elements of the two partitions. The maximal element is $(\{1,2, \ldots, n\})$, and the minimal element is $(\{1\},\{2\}, \ldots,\{n\})$. 
(For the corresponding result for unordered partitions see [5, Lemma 1, p.192] and [2, Theorem 6, p.7]). It is easy to see that the greatest lower bound, $\Theta=\left(\theta_{1}, \ldots, \theta_{q}\right)$, of the set of partitions for which $X$ is block lower triangular is also a partition for which $X$ is block lower triangular, and we will refer to $\Theta$ as the block lower triangular self-partition of $X$. Similarly, the greatest lower bound, $\Phi=\left(\phi_{1}, \ldots, \phi_{p}\right)$, of the partitions $\Upsilon=\left(v_{1}, v_{2}, \ldots, v_{r}\right)$, for which $X_{v_{i}^{+} v_{i}}=0$ is referred to as the block upper triangular self-partition of $X$. Thus we say $X$ is lower triangular if the block lower triangular self-partition of $X$ is $(\{1\},\{2\} \ldots\{n\})$. Similarly, we say $X$ is upper triangular if the block upper triangular self-partition of $X$ is $(\{1\},\{2\} \ldots\{n\})$.

To illustrate the definitions above, let

$$
X=\left[\begin{array}{cccccccc}
* * & * & 0 & 0 & 0 & 0 & 0 \\
0 & * & 0 & 0 & 0 & 0 & 0 & 0 \\
0 & 0 & * & 0 & 0 & 0 & 0 & 0 \\
0 & 0 & 0 & * & 0 & 0 & * & 0 \\
0 & 0 & 0 & 0 & 0 & * & 0 & 0 \\
0 & 0 & 0 & 0 & 0 & 0 & 0 & 0 \\
0 & 0 & 0 & 0 & * & 0 & * & 0 \\
0 & 0 & 0 & 0 & 0 & 0 & 0 & *
\end{array}\right],
$$

where $*$ denotes a nonzero entry. Then viewed as a block lower triangular matrix

$$
X=\left[\begin{array}{ccc|ccccc}
* & * & * & 0 & 0 & 0 & 0 & 0 \\
0 & * & 0 & 0 & 0 & 0 & 0 & 0 \\
0 & 0 & * & 0 & 0 & 0 & 0 & 0 \\
\hline 0 & 0 & 0 & * & 0 & 0 & * & 0 \\
0 & 0 & 0 & 0 & 0 & * & 0 & 0 \\
0 & 0 & 0 & 0 & 0 & 0 & 0 & 0 \\
0 & 0 & 0 & 0 & * & 0 & * & 0 \\
0 & 0 & 0 & 0 & 0 & 0 & 0 & 0
\end{array}\right],
$$

and has block lower triangular self-partition $(\{1,2,3\},\{4,5,6,7\},\{8\})$. However, viewed as a block upper triangular matrix

$$
X=\left[\begin{array}{cccccccc}
* & * & * & 0 & 0 & 0 & 0 & 0 \\
\hline 0 & * & 0 & 0 & 0 & 0 & 0 & 0 \\
0 & 0 & * & 0 & 0 & 0 & 0 & 0 \\
0 & 0 & 0 & * & 0 & 0 & * & 0 \\
0 & 0 & 0 & 0 & 0 & * & 0 & 0 \\
0 & 0 & 0 & 0 & 0 & 0 & 0 & 0 \\
0 & 0 & 0 & 0 & * & 0 & * & 0 \\
0 & 0 & 0 & 0 & 0 & 0 & 0 & 0
\end{array}\right],
$$


and has block upper triangular self-partition

$$
(\{1\},\{2\},\{3\},\{4\},\{5,6,7\},\{8\}) .
$$

Let $J_{1}, \ldots, J_{r}$ be subsets of $\langle n\rangle$. We say that an order preserving partition $\Psi=\left(\psi_{1}, \ldots, \psi_{t}\right)$, encompasses $J_{1}, \ldots, J_{r}$, if for each $i \in\langle r\rangle$, there exists $k \in\langle t\rangle$ such that $J_{i} \subseteq \psi_{k}$. It is easy to see that the greatest lower bound of the order preserving partitions which encompass $J_{1}, \ldots, J_{r}$, also encompasses $J_{1}, \ldots, J_{r}$ and we will refer to this as the finest order preserving partition encompassing $J_{1}, \ldots, J_{r}$.

Next we define subsets $T_{i}$ and $F_{i}$ associated with the matrix $A$. These subsets are defined in terms of the access relationships in $\mathcal{G}(A)$ to and from the singular classes of $A$, and are convex in the sense that if $j, k \in T_{i}$ (or $\left.F_{i}\right)$, then $l \in T_{i}\left(F_{i}\right)$ for every $j \leq l \leq k$.

Definition 2.1 Let $A \in \mathbb{R}^{n n}$. Let $S_{1}, S_{2}, \ldots S_{m}$ be the singular classes of $A$ ordered so that $\max \left(S_{i}\right)<\max \left(S_{i+1}\right)$. For each $i \in\langle m\rangle$, let $\mu_{i}=$ $\max \left(S_{i}\right)$, and

$$
\begin{aligned}
F_{i}= & \left\{l \geq \mu_{i} \mid \text { there exists } j \geq l \text { such that } j \text { is accessed FROM } S_{i}\right. \\
& \text { in } \mathcal{G}(A)\} . \\
T_{i}= & \left\{l \geq \mu_{i} \mid \text { there exists } j \geq l \text { such that } j \text { has access TO } S_{i} \text { in } \mathcal{G}(A)\right\},
\end{aligned}
$$

These subsets turn out to be the key to understanding the block structure of a block LU factorization of $A$.

Notice that the $F_{i}$ for $A$ correspond to the $T_{i}$ for $A^{\mathrm{T}}$ and vice versa.

Remark 2.2 In [13], Varga and Cai show that an M-matrix admits an LU factorization into M-matrices with $L$ nonsingular if and only if $T_{i}=\left\{\mu_{i}\right\}$ for every $i \in\langle m\rangle$.

Notice that if $j>i$ and vertices in $S_{j}$ are accessed from vertices in $S_{i}$, then $F_{j} \subset F_{i}$, so if $i$ is placed in $J$, then $j$ should be also. Similarly if vertices in $S_{j}$ have access to vertices in $S_{i}$, then $T_{j} \subset T_{i}$ and hence if $i$ is placed in $K$, then $j$ should be also.

In Theorem 3.5 we show that for each $i \in\langle m\rangle$, either $F_{i}$ is encompassed in the block lower triangular self-partition of $L$ or $T_{i}$ is encompassed in the block upper triangular self-partition of $U$. This suggests that when factoring an M-matrix $A$, these $T_{i}$ and $F_{i}$ should be examined in order to determine an optimal factorization of a given type. There are several possibilities for the types of factorizations one might want. In this paper we highlight four possibilities. In Strategy 3.10 we outline a strategy for choosing a partition $(J, K)$ so as to minimize the sizes of the blocks in a block LU factorization of $A$. In Strategy 3.11 we outline a strategy for choosing a permutation 
matrix $P$ so that $P A P^{\mathrm{T}}$ has an LU factorization. In Sect. 4 we look at the structure of $U$ if $L$ is required to be nonsingular (or vice versa). In Sect. 5, we look at factoring $A$ as $L B U$ where $L$ is a nonsingluar lower triangular $\mathrm{M}$-matrix, $U$ is a nonsingular upper trianglular $\mathbf{M}$-matrix, and $B$ is block diagonal.

Example 2.3 Here we provide an example which illustrates the definitions introduced above.

Let

$$
A=\left[\begin{array}{cccc}
0 & -1 & 0 & 0 \\
0 & 0 & 0 & -1 \\
0 & -1 & 0 & 0 \\
0 & 0 & 0 & 1
\end{array}\right] .
$$

Then the block lower triangular self-partition of $A$ is $(\{1,2,3,4\})$, and the block upper triangular self-partition is $(\{1\},\{2,3\},\{4\})$. The singular classes of $A$ are

$$
S_{1}=\{1\}, S_{2}=\{2\}, S_{3}=\{3\},
$$

and

$$
\begin{gathered}
T_{1}=\{1\}, T_{2}=\{2,3\}, T_{3}=\{3\}, \\
F_{1}=\{1,2,3,4\}, F_{2}=\{2,3,4\}, F_{3}=\{3\} .
\end{gathered}
$$

Notice that $T_{3} \subseteq T_{2}$ and $F_{3} \subseteq F_{2} \subseteq F_{1}$.

In Sect. 4, we expand the results of [13] to include a larger set of LU factorizations, and we examine the block structure when no LU factorization exists. The class associated with an $\mathrm{M}$-matrix $A$ defined next includes all of the matrices $U$ for which $A=L U$, with $L, U \in \mathcal{M}$ and $L$ nonsingular. Notice that this is actually a larger class since it allows for some M-matrices $U \in \mathcal{M}$ for which $A=L U$ with $L$ inverse nonnegative but not necessarily an M-matrix.

For any $A \in \mathbb{R}^{n n}$, we say a matrix $X$ is class nonsingular for $A$ if for every class $K$ of $A, X_{K K}$ is nonsingular (see [9]). Note that $K$ need not be a class of $X$. We write

$$
\mathcal{N}_{A}=\{X \mid X \geq 0 \text { and } X \text { is class nonsingular for } A\} .
$$

Definition 2.4 Let $A$ be an M-matrix and define

$$
\begin{aligned}
& \mathcal{U}(A)=\left\{B A \mid B \in \mathcal{N}_{A}, B A \in \mathcal{M}\right\} . \\
& \mathcal{L}(A)=\left\{A B \mid B \in \mathcal{N}_{A}, A B \in \mathcal{M}\right\} .
\end{aligned}
$$




\section{Block LU factorizations with (possibly) singular $L$ and $U$.}

In [13], Varga and Cai consider LU factorizations of M-matrices where $\mathrm{L}$ is nonsingular (see Remark 2.2). In Example 3.1, the matrix $A$ does not satisfy the conditions stated in [13] and hence does not have a LU factorization into $\mathrm{M}$-matrices with $L$ is nonsingular. It does however, have an LU factorization when both $L$ and $U$ are allowed to be singular.

Example 3.1 Let

$$
A=\left[\begin{array}{rrr}
0 & -1 & 0 \\
0 & 0 & 0 \\
0 & -1 & 0
\end{array}\right]
$$

By [13, Theorem 1,(see Remark 2.2)], $A$ does not admit a factorization $A=L U$ with $L$ a nonsingular lower triangular M-matrix and $U$ an upper triangular $\mathrm{M}-$ matrix. By applying the result to $A^{\mathrm{T}}$ we see that $A$ does not admit a factorization $A=L U$ with $L$ a lower triangular M-matrix and $U$ a nonsingular upper triangular $\mathrm{M}$-matrix. However, consider

$$
L=\left[\begin{array}{rrr}
1 & 0 & 0 \\
0 & 0 & 0 \\
0 & -1 & 0
\end{array}\right], \quad U=\left[\begin{array}{rrr}
0 & -1 & 0 \\
0 & 1 & 0 \\
0 & 0 & 0
\end{array}\right]
$$

Then $A=L U$, where $L$ is a singular lower triangular $\mathrm{M}$-matrix and $U$ is a singular upper triangular M-matrix.

In this section we establish necessary and sufficient conditions for $A$ to have an LU factorization into M-matrices, allowing both $L$ and $U$ to be singular. This result is stated as Corollary 3.9. Enroute to establishing this result we also characterize the minimum block structure of block LU factorizations when no triangular factorization exists. These results appear as Theorem 3.5 and Theorem 3.7.

In Strategy 3.10, we outline a strategy one might take to minimize the block sizes in a block LU factorization of $A$. In Strategy 3.11, we suggest a permutation $P$ such that $P A P^{\mathrm{T}}$ admits an LU factorization.

We begin with two lemmas and a corollary which we use to prove the main results in this section (Theorem 3.5, Theorem 3.7 and Corollary 3.9).

First we show that if $A=L U$ is a factorization of an M-matrix $A$ into M-matrices and $S$ is a singular class of $L$, then the vertices which are accessed by any vertex of $S$ in $\mathcal{G}(A)$, are also accessed by the vertices of $S$ in $\mathcal{G}(L)$. 
Lemma 3.2 Let $A \in \mathcal{M}$ with factorization $A=L U$ where $L, U \in \mathcal{M}$. Let $S$ be any singular class of $L$. Then for any $p \in S$ and any $q \in\langle n\rangle$, if $p$ has access to $q$ in $\mathcal{G}(A)$ then $p$ has access to $q$ in $\mathcal{G}(L)$.

Proof. Let $q \in\langle n\rangle$. Suppose some $p \in S$ has access to $q$ in $\mathcal{G}(A)$ but not in $\mathcal{G}(L)$. Then by choosing an appropriate section of a path from $p$ to $q$ in $\mathcal{G}(A)$, we can choose a path $l_{1} \rightarrow l_{2} \rightarrow \ldots \rightarrow l_{g}$, in $\mathcal{G}(A)$ so that

$$
l_{1} \in S,
$$

$l_{1}$ has access to $l_{i}$ in $\mathcal{G}(L)$ and $l_{i} \notin S$ for all $i=2, \ldots, g-1$

$l_{1}$ does not have access to $l_{g}$ in $\mathcal{G}(L)$,

$$
l_{i} \neq l_{j} \text {, for all } i \neq j \text {. }
$$

We will establish a contradiction by proving two claims. The proofs of Claim 1 and Claim 2 rely heavily on the sign patterns of $A, L$, and $U$.

Claim 1. If $r \in S, t \notin S$, are such that $L_{r S} U_{S t}<0$, then there exists $s \in S$ such that $L_{s t} \neq 0$.

Proof of Claim 1. Since $L_{S S}$ is an irreducible singular M-matrix and $L_{S S} U_{S t} \neq 0$, by [1, Theorem 4.16, p. 156] it must be a vector which contains both positive and negative elements. Hence there exists $s \in S$ such that $L_{s S} U_{S t}>0$. But then $0 \geq A_{s t}=L_{s t} U_{t t}+P$, where $P>0$. Hence $L_{s t} U_{t t}<0$, and in particular $L_{s t} \neq 0$. This establishes Claim 1.

Claim 2. For each $j \in\langle g-1\rangle$,

(a) There exists $r \in S \cup\left\{l_{2}, \ldots, l_{j}\right\}$ with $L_{r l_{j+1}} \neq 0$,

(b) If $\notin S \cup\left\{l_{2}, \ldots, l_{j+1}\right\}$ and $U_{l_{j+1} t} \neq 0$, then there exists $r \in S \cup\left\{l_{2}, \ldots, l_{j}\right\}$ with $L_{r t} \neq 0$,

Proof of Claim 2. We proceed by induction on $j$.

Let $j=1$ :

(a) Since $0>A_{l_{1} l_{2}}=L_{l_{1} l_{2}} U_{l_{2} l_{2}}+L_{l_{1} S} U_{S l_{2}}+P$, where $P \geq 0$, either $L_{l_{1} l_{2}} U_{l_{2} l_{2}}<0$ and thus $L_{l_{1} l_{2}} \neq 0$, or $L_{l_{1} S} U_{S l_{2}}<0$ and thus by Claim 1 there exists $s \in S$ such that $L_{s l_{2}} \neq 0$.

(b) Suppose $U_{l_{2} t} \neq 0$, for some $t \notin S \cup\left\{l_{2}\right\}$. By (a) there exists $r \in S$ with $L_{r l_{2}} \neq 0$, hence $L_{r l_{2}} U_{l_{2} t}>0$. Thus $0 \geq A_{r t}=$ $L_{r t} U_{t t}+L_{r S} U_{S t}+P$, where $P>0$. Hence either $L_{r t} U_{t t}<0$ and thus $L_{r t} \neq 0$, or $L_{r S} U_{S t}<0$ and by Claim 1, there exists $s \in S$ such that $L_{s t} \neq 0$. 
Let $k<g$. Assume true for all $j$ with $1 \leq j<k$ and show true for $k$.

(a) Since $0>A_{l_{k} l_{k+1}}=L_{l_{k} l_{k+1}} U_{l_{k+1} l_{k+1}}+L_{l_{k} l_{k}} U_{l_{k} l_{k+1}}+P$, where $P \geq 0$, either $L_{l_{k} l_{k+1}} U_{l_{k+1} l_{k+1}}<0$ and thus $L_{l_{k} l_{k+1}} \neq 0$, or $L_{l_{k} l_{k}} U_{l_{k} l_{k+1}}<0$ and so by the induction hypothesis (b) applied with $j=k-1$ and $t=l_{k+1}$, there exists $r \in S \cup\left\{l_{2}, \ldots, l_{k-1}\right\}$ such that $L_{r l_{k+1}} \neq 0$.

(b) Suppose $U_{l_{k+1} t} \neq 0$, for some $t \notin S \cup\left\{l_{2}, \ldots, l_{k+1}\right\}$. By (a) there exists $r \in S \cup\left\{l_{2}, \ldots, l_{k}\right\}$ such that $L_{r l_{k+1}} \neq 0$, hence $L_{r l_{k+1}} U_{l_{k+1} t}>0$. Thus $0 \geq A_{r t}=L_{r t} U_{t t}+L_{r r} U_{r t}+P$, where $P>0$. Hence either $L_{r t} U_{t t}<0$ and thus $L_{r t} \neq 0$, or $L_{r r} U_{r t}<0$ and thus $U_{r t}<0$ and so by the induction hypothesis applied with $j=k-1$, there exists $q \in S \cup\left\{l_{2}, \ldots, l_{k-1}\right\}$ with $L_{q t} \neq 0$.

This establishes Claim 2.

By Claim 2, there exists $r \in S \cup\left\{l_{2}, \ldots, l_{g-1}\right\}$ such that $L_{r l_{g}} \neq 0$, but then there exists $t \in S$ such that $t$ has access to $l_{g}$ in $\mathcal{G}(L)$, and since $S$ is a class of $\mathcal{G}(L)$, it must be that $l_{1}$ has access to $l_{g}$ in $\mathcal{G}(L)$. Contradiction. Hence $p$ must have access to $q$ in $\mathcal{G}(L)$.

By applying Lemma 3.2 to $A^{\mathrm{T}}$ we get the following result.

Corollary 3.3 Let $A \in \mathcal{M}$ with factorization $A=L U$ where $L, U \in \mathcal{M}$. Let $S$ be any singular class of $U$. Then for any $p \in S$ and any $q \in\langle n\rangle$, if $p$ is accessed by $q$ in $\mathcal{G}(A)$ then $p$ is accessed by $q$ in $\mathcal{G}(U)$.

Proof. Apply Lemma 3.2 to $A^{\mathrm{T}}=U^{\mathrm{T}} L^{\mathrm{T}}$.

Next we show that every singular class of $A$ has a corresponding singular class $Q \subseteq S$ in either $L$ or $U$.

Lemma 3.4 Let $A \in \mathcal{M}$ with factorization $A=L U$ where $L, U \in \mathcal{M}$. Let $S$ be any singular class of $A$. Then either $L_{S S}$ is singular or $U_{S S}$ is singular, and there exists $Q \subseteq S$ such that $Q$ is a singular class of either $L$ or $U$.

Proof.

$$
A_{S S}=L_{S S} U_{S S}+L_{S S^{\prime}} U_{S^{\prime} S}=L_{S S} U_{S S}+P \text {, where } P \geq 0 \text {. }
$$

Thus $L_{S S} U_{S S}=A_{S S}-P$ and hence is a Z-matrix. Suppose that $L_{S S}$ and $U_{S S}$ are both nonsingular. Then by [1, $N_{44}$, p. 137], $L_{S S} U_{S S}$ is a nonsingular M-matrix, and by $\left[1, I_{27}, \mathrm{p} .137\right]$ there exists $x \gg 0$ such that $L_{S S} U_{S S} x \gg 0$. But then $A_{S S} x=L_{S S} U_{S S} x+P x \gg 0$, which implies that $A_{S S}$ is a nonsingular M-matrix. A contradiction. Hence either $L_{S S}$ is singular or $U_{S S}$ is singular. Since any proper principal submatrix of an 
irreducible M-matrix is nonsingular, it must be the case that if $L_{S S}$ (or $U_{S S}$ ) is singular, then the singular class $Q$ of $L_{S S}$ (or $U_{S S}$ ) is a singular class of $L$ (or $U$ ).

The next theorem shows that certain access relationships must be present either in $L$ or $U$. In particular, for each $i \in\langle m\rangle$, either $F_{i}$ is encompassed in a block of $L$ or $T_{i}$ is encompassed in a block of $U$.

Theorem 3.5 (Minimality) Let $A \in \mathcal{M}$ with factorization $A=L U$ where $L, U \in \mathcal{M}$. Let $S_{i}, T_{i}, F_{i}$, and $m$ be as in Definition 2.1. Then there is $a Q_{i} \subseteq S_{i}$ such that $Q_{i}$ is a singular class of either $L$ or $U$. If $Q_{i}$ is a singular class of $L$, then $F_{i}$ is encompassed in the block lower triangular self-partition of $L$. If $Q_{i}$ is a singular class of $U$, then $T_{i}$ is encompassed in the block upper triangular self-partition of $U$.

Proof. That there exists such a $Q_{i}$ follows directly from Lemma 3.4. If $Q_{i}$ is a singular class of $L$ then by Lemma 3.2, any $j$ which is accessed from $S_{i}$ (and hence $Q_{i}$ ) in $\mathcal{G}(A)$ is also accessed from $Q_{i}$ in $\mathcal{G}(L)$, thus $F_{i}$ is encompassed in the block lower triangular self-partition of $L$. Similarly, if $Q_{i}$ is a singular class of $U$, then Corollary 3.3 implies that $T_{i}$ is encompassed in the block upper triangular self-partition of $U$.

It is natural for one to ask if, once an assignment of the singular classes between $L$ and $U$ has been chosen, such factorizations can be achieved. The following example shows that the singular classes of $A$ cannot necessarily be divided up between $L$ and $U$ to suit ones choosing.

Example 3.6 Let

$$
A=\left[\begin{array}{cc}
0 & -1 \\
0 & 0
\end{array}\right] .
$$

The singular classes of $A$ are $S_{1}=\{1\}$ and $S_{2}=\{2\}$. It is easy to verify that there is no factorization $A=L U$ where $S_{1}$ is a singular class of $L$ and $S_{2}$ is a singular class of $U$. Notice that $F_{2} \subset F_{1}$ and hence there is no combinatorial benefit to having $S_{2}$ not be a singular class of $L$ once $S_{1}$ has been chosen to be a singular class of $L$.

In the next theorem we show that once a partition $(J, K)$ of $\langle m\rangle$ has been chosen, a block factorization can be achieved with the block structure of $L$ being a refinement of the partition encompassing $F_{i}, i \in J$ and the block structure of $U$ being a refinement of the partition encompassing $T_{i}, i \in K$.

Theorem 3.7 (Existence) Let $A \in \mathcal{M}$. Let $S_{i}, T_{i}, F_{i}$, and $m$ be as in Definition 2.1. Let $(J, K)$ be a partition of $\langle m\rangle$. Let $\Psi$ be the finest order 
preserving partition of $\langle n\rangle$ encompassing $F_{i}, i \in J$ and let $\Upsilon$ be the finest order preserving partition of $\langle n\rangle$ encompassing $T_{i}, i \in K$. Then there exists a factorization $A=L U$ such that $L, U \in \mathcal{M}$, the block lower triangular self-partition of $L$ is a refinement of $\Psi$, and the block upper triangular selfpartition of $U$ is a refinement of $Y$.

Proof. We establish this result by outlining a recursive algorithm which gives us the desired factorization.

If $a_{11} \neq 0$ then let

$$
N=\{2, \ldots, n\}
$$

and set

$$
B=A_{N N}-\frac{1}{a_{11}} A_{N 1} A_{1 N} .
$$

Notice that $B$ is an M-matrix and $\mathcal{G}(B) \subseteq \Gamma$ where $\Gamma$ is the subgraph of $\overline{\mathcal{G}(A)}$ induced by the vertices $2, \ldots, n$. Moreover, if $Q$ is a singular class of $B$ (where $B$ is indexed with indices corresponding to $A$ ) then $Q \subseteq S$ where $S$ is a singular class of $A$. Now apply the algorithm to $B$ to obtain $B=\hat{L} \hat{U}$ where $\hat{L}$ and $\hat{U}$ satisfy the theorem. Set

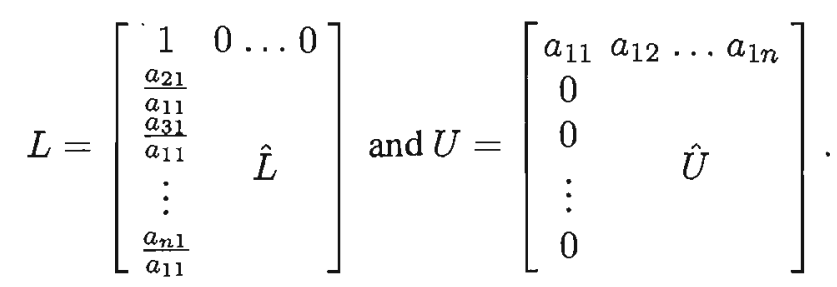

Then $A=L U$ satisfies the theorem.

If $a_{11}=0$, then $1=\mu_{1}$.

(i) If $1 \in J$ then set

$$
\begin{gathered}
V=\left\{l \mid \mu_{1} \text { has access to } l \text { in } \mathcal{G}(A),\right. \\
W=\langle n\rangle \backslash V
\end{gathered}
$$

Notice that $A_{V W}=0, V \subseteq F_{1}$, and $\max (V)=\max \left(F_{1}\right)$. Choose a permutation matrix $P$ such that

$$
P^{-1} A P=\left[\begin{array}{cc}
A_{V V} & 0 \\
A_{W V} & A_{W W}
\end{array}\right]=\left[\begin{array}{cc}
A_{V V} & 0 \\
A_{W V} & I
\end{array}\right]\left[\begin{array}{cc}
I & 0 \\
0 & A_{W W}
\end{array}\right],
$$

where elements of $V$ and $W$ are listed in ascending order. Since $A_{W W}$ is a principal submatrix of $A$ it is also an $\mathrm{M}$-matrix and we can apply the algorithm to $A_{W W}$ to get $A_{W W}=\hat{L} \hat{U}$ where $\hat{L}$ and $\hat{U}$ have the desired structure based on the properties of $A_{W W}$. Set

$$
L=P\left[\begin{array}{ll}
A_{V V} & 0 \\
A_{W V} & \hat{L}
\end{array}\right] P^{-1}, \quad U=P\left[\begin{array}{cc}
I & 0 \\
0 & \hat{U}
\end{array}\right] P^{-1} .
$$


Then $A=L U$. We now argue that this factorization satisfies the theorem. Since applying the permutation similarity only reorders vertices, we see that $L_{V W}=0$ and $L_{W W}=\hat{L}$ where $\hat{L}$ has the desired block structure based on the properties of $A_{W W}$. We also have that $V \subset F_{1}$, thus the block lower triangular self-partition of $L$ is a refinement of $\Psi$. By again observing that applying the permutation similarity with $P$ merely reorders vertices, we see that $U_{V V}$ is a diagonal matrix, $U_{V W}=0, U_{W V}=0$, and $U_{W W}=\hat{U}$ has the desired structure based on the properties of $A_{W W}$. Thus the block upper triangular self-partition of $U$ is a refinement of $\Upsilon$.

(ii) If $1 \notin J$ then $1 \in K$, and we can apply the algorithm to $A^{\mathrm{T}}$ with $J$ and $K$ interchanged to get $A^{\mathrm{T}}=\hat{L} \hat{U}$. Set $L=\hat{U}^{\mathrm{T}}$ and $U=\hat{L}^{\mathrm{T}}$. Since transposing a matrix reverses the access relationships, the $F_{i}$ for $A$ correspond to the $T_{i}$ for $A^{\mathrm{T}}$ and vice versa. By the argument presented in $(i)$, the factorization $A=L U$ has the desired properties.

Example 3.8 Let

$$
A=\left[\begin{array}{rrr}
0 & -1 & 0 \\
0 & 0 & 0 \\
0 & -1 & 0
\end{array}\right] .
$$

Let $J=\{1\}$ and $K=\{2,3\}$. Then $(J, K)$ is a partition of the singular classes of $A$, however since $F_{2} \subset F_{1}, S_{2}$ is automatically a singular class of $L$, thus $T_{2}$, as the following factorization shows, need not be encompassed in the block structure of $U$. Notice that

$$
L=\left[\begin{array}{rrr}
0 & -1 & 0 \\
0 & 0 & 0 \\
0 & -1 & 1
\end{array}\right], \quad U=\left[\begin{array}{lll}
1 & 0 & 0 \\
0 & 1 & 0 \\
0 & 0 & 0
\end{array}\right]
$$

provides an LU factorization which is a proper refinement of the partition identified by Theorem 3.7. Here $S_{2}$ is a singular class of $L$ rather than of $U$.

The partitioning of the singular classes between $L$ and $U$ of this factorization is actually $J=\{1,2\}, K=\{3\}$. Using this paritition of the singular classes, Theorem 3.5 and Theorem 3.7 guarantee that the $L$ and the $U$ listed above have the smallest possible blocks for this assignment of the singular classes between $L$ and $U$.

Next we state necessary and sufficient conditions for an M-matrix $A$ to admit an LU factorization into $\mathrm{M}$-matrices, thus extending the results in [13] to the case where both $L$ and $U$ are allowed to be singular.

Corollary 3.9 Let $A \in \mathcal{M}$. Then the following are equivalent: 
(i) A admits a factorization $A=L U$, where $L$ is a lower triangular $M-$ matrix and $U$ is a upper triangular $M$-matrix.

(ii) Let $S_{i}, T_{i}, F_{i}$, and $m$ be as in Definition 2.1. Then for every $i \in\langle m\rangle$, either $T_{i}=\left\{\mu_{i}\right\}$ or $F_{i}=\left\{\mu_{i}\right\}$.

Strategy 3.10 Using Theorem 3.5 and Theorem 3.7 one can strategically choose, for example, a partition which minimizes the sizes of the blocks in $L$ and $U$. Let $S_{i}, \mu_{i}, T_{i}, F_{i}$, and $m$ be as in Definition 2.1. For each $i \in\langle m\rangle$, either $F_{i}$ has to be encompassed in block of $L$ or $T_{i}$ has to be encompassed in a block of $U$. In each case, we choose the smallest block between the two, unless one of the sets $\left(T_{i}\right.$ or $\left.F_{i}\right)$ is a subset of an earlier choosen set in which case it has already been taken care of.

Begin by setting $V=\langle m\rangle$.

(i) For $i=\min (V)$, if $\left|F_{i}\right|<\left|T_{i}\right|$, put $i$ into $J$, otherwise put $i \in K$. Remove $i$ from $V$. At this time other elements from $V$ may have automatically been taken care of (see Example 3.8). Hence if $i$ was placed in $J$, then for each $j \in V$ such that $F_{j} \subseteq F_{i}$, place $j$ in $J$ and remove $j$ from $V$. If $i$ was placed in $K$, then for each $j \in V$ such that $T_{j} \subseteq T_{i}$, place $j$ in $K$ and remove $j$ from $V$.

(ii) Repeat (i) until $V=\emptyset$.

(iii) Apply the algorithm provided in the proof of Theorem 3.7 with the partition $(J, K)$.

Strategy 3.11 Another strategy one might employ is to choose a permutation matrix $P$ such that $P A P^{\mathrm{T}}$ satisfies Corollary 3.7 , and hence has an LU factorization. There are several ways one might do this. For example, using $\mu_{i}$ and $m$ as in Definition 2.1, one could choose a permutation matrix $P$ which corresponds to the permutation which moves $\mu_{1}, \ldots \mu_{m}$ to positions $n-m+1, \ldots n$ and reorders them (if necessary) so that $\mu_{i}$ is placed after $\mu_{j}$ whenever $\mu_{i}$ has access to $\mu_{j}$ in $\mathcal{G}(A)$. All other indices should be shifted up by the appropriate amount. Then $F_{i}=\left\{\mu_{i}\right\}$ so by Corollary 3.9 , a triangular LU factorization exists with $J=\langle m\rangle$ and $K=\emptyset$.

We conclude this section with two examples on which we illustrate the strategies suggested by the theorems in this section.

Example 3.12 Let

$$
A=\left[\begin{array}{ccccccc}
1 & -1 & 0 & 0 & -1 & 0 & 0 \\
-1 & 1 & 0 & 0 & -2 & 0 & 0 \\
0 & 0 & 2 & -2 & 0 & 0 & 0 \\
0 & 0 & -2 & 2 & 0 & 0 & 0 \\
0 & 0 & 0 & 0 & 1 & 0 & 0 \\
0 & 0 & -1 & -1 & 0 & 0 & 0 \\
0 & 0 & 0 & 0 & 0 & -1 & 1
\end{array}\right]
$$


Let $S_{i}, \mu_{i}, T_{i}, F_{i}$ be as in Definition 2.1. Then

$$
\begin{gathered}
S_{1}=\{1,2\}, S_{2}=\{3,4\}, S_{3}=\{6\}, \\
\mu_{1}=2, \mu_{2}=4, \mu_{3}=6, \\
T_{1}=\{2\}, T_{2}=\{4,5,6,7\}, T_{3}=\{6,7\}, \\
F_{1}=\{2,3,4,5\}, F_{2}=\{4\}, F_{3}=\{6\} .
\end{gathered}
$$

Thus by [13, Theorem 1] (see Remark 2.2) $A$ does not admit an LU factorization into M-matrices with $L$ nonsingular. Similarly by [13, Theorem 1] applied to $A^{\mathrm{T}}, A$ does not admit an LU factorization into M-matrices with $U$ nonsingular. Using Strategy 3.10 we choose $J=\{2,3\}$ and $K=\{1\}$. The algorithm outlined in the proof of Theorem 3.7 now gives us an LU factorization with

$$
L=\left[\begin{array}{ccccccc}
1 & 0 & 0 & 0 & 0 & 0 & 0 \\
-1 & 1 & 0 & 0 & 0 & 0 & 0 \\
0 & 0 & 2 & 0 & 0 & 0 & 0 \\
0 & 0 & -2 & 0 & 0 & 0 & 0 \\
0 & 0 & 0 & 0 & 1 & 0 & 0 \\
0 & 0 & -1 & -2 & 0 & 0 & 0 \\
0 & 0 & 0 & 0 & 0 & -1 & 1
\end{array}\right], U=\left[\begin{array}{ccccccc}
1 & -1 & 0 & 0 & -1 & 0 & 0 \\
0 & 0 & 0 & 0 & -3 & 0 & 0 \\
0 & 0 & 1 & -1 & 0 & 0 & 0 \\
0 & 0 & 0 & 1 & 0 & 0 & 0 \\
0 & 0 & 0 & 0 & 1 & 0 & 0 \\
0 & 0 & 0 & 0 & 0 & 1 & 0 \\
0 & 0 & 0 & 0 & 0 & 0 & 1
\end{array}\right]
$$

Example 3.13 Let

$$
A=\left[\begin{array}{cccccccc}
1 & -1 & 0 & 0 & 0 & 0 & 0 & 0 \\
0 & 0 & -1 & 0 & 0 & 0 & 0 & 0 \\
0 & 0 & 1 & 0 & -1 & 0 & 0 & 0 \\
0 & 0 & 0 & 1 & 0 & 0 & 0 & 0 \\
0 & 0 & -1 & 0 & 1 & -1 & 0 & 0 \\
0 & 0 & 0 & 0 & 0 & 0 & 0 & 0 \\
0 & 0 & 0 & 0 & 0 & 0 & 0 & -1 \\
-1 & 0 & 0 & 0 & 0 & 0 & 0 & 0
\end{array}\right]
$$

Let $S_{i}, \mu_{i}, \mathcal{T}_{i}, F_{i}$ be as in Definition 2.1. Then

$$
\begin{gathered}
S_{1}=\{2\}, S_{2}=\{3,5\}, S_{3}=\{6\}, S_{4}=\{7\}, S_{5}=\{8\}, \\
\mu_{1}=2, \mu_{2}=5, \mu_{3}=6, \mu_{4}=7, \mu_{5}=8, \\
F_{1}=\{2,3,4,5,6\}, F_{2}=\{5,6\}, F_{3}=\{6\}, F_{4}=\{7,8\}, F_{5}=\{8\}, \\
T_{1}=\{1,2,3,4,5,6,7,8\}, T_{2}=\{5,6,7,8\}, T_{3}=\{6,7,8\}, \\
\mathcal{T}_{4}=\{7\}, T_{5}=\{8\} .
\end{gathered}
$$


Using strategy 3.10 one should set $J=\{1,2,3\}$, and $K=\{4,5\}$. Using the algorithm outlined in Theorem 3.7 we get

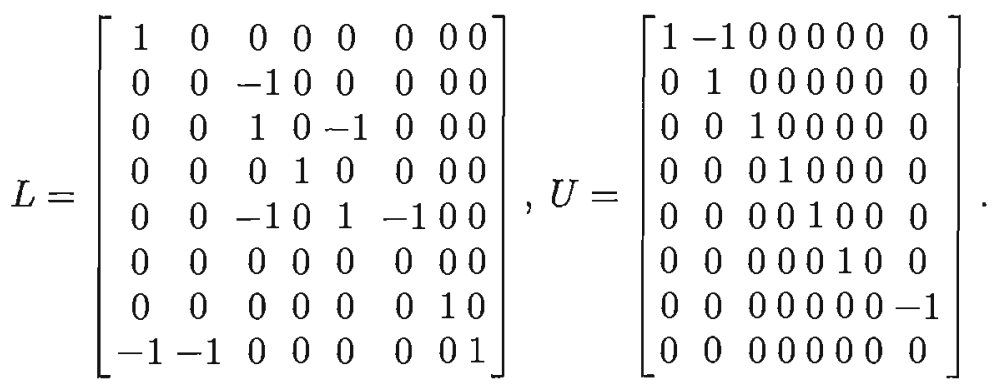

This factorization minimizes the sizes of the blocks in $L$ and $U$.

Using strategy 3.11 , we can choose a permutation which moves $2,5,6$, 7,8 to the end of our list of indices and then reorders them as $6,5,2,8,7$. Thus we take

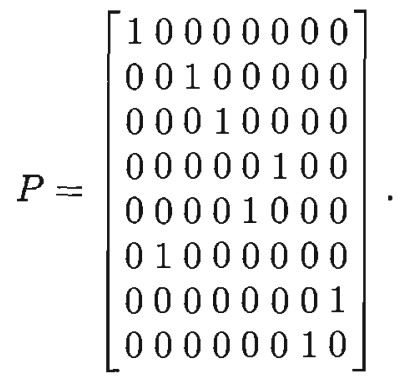

Then

$$
P A P^{-1}=\left[\begin{array}{cccccccc}
1 & 0 & 0 & 0 & 0 & -1 & 0 & 0 \\
0 & 1 & 0 & 0 & -1 & 0 & 0 & 0 \\
0 & 0 & 1 & 0 & 0 & 0 & 0 & 0 \\
0 & 0 & 0 & 0 & 0 & 0 & 0 & 0 \\
0 & -1 & 0 & -1 & 1 & 0 & 0 & 0 \\
0 & -1 & 0 & 0 & 0 & 0 & 0 & 0 \\
-1 & 0 & 0 & 0 & 0 & 0 & 0 & 0 \\
0 & 0 & 0 & 0 & 0 & 0 & -1 & 0
\end{array}\right]
$$

which has an LU factorization with

$$
L=\left[\begin{array}{cccccccc}
1 & 0 & 0 & 0 & 0 & 0 & 0 & 0 \\
0 & 1 & 0 & 0 & 0 & 0 & 0 & 0 \\
0 & 0 & 1 & 0 & 0 & 0 & 0 & 0 \\
0 & 0 & 0 & 0 & 0 & 0 & 0 & 0 \\
0 & -1 & 0 & -1 & 0 & 0 & 0 & 0 \\
0 & -1 & 0 & 0 & -1 & 0 & 0 & 0 \\
-1 & 0 & 0 & 0 & 0 & -1 & 0 & 0 \\
0 & 0 & 0 & 0 & 0 & 0 & -1 & 0
\end{array}\right], U=\left[\begin{array}{cccccccc}
1 & 0 & 0 & 0 & 0 & -1 & 0 & 0 \\
0 & 1 & 0 & 0 & -1 & 0 & 0 & 0 \\
0 & 0 & 1 & 0 & 0 & 0 & 0 & 0 \\
0 & 0 & 0 & 1 & 0 & 0 & 0 & 0 \\
0 & 0 & 0 & 0 & 1 & 0 & 0 & 0 \\
0 & 0 & 0 & 0 & 0 & 1 & 0 & 0 \\
0 & 0 & 0 & 0 & 0 & 0 & 1 & 0 \\
0 & 0 & 0 & 0 & 0 & 0 & 0 & 1
\end{array}\right]
$$




\section{Block $L U$ factorizations with nonsingular $L$}

In this section we consider the case where an M-matrix $A$ is factored into block triangular matrices with $L$ nonsingular, such that $L^{-1}$ is nonnegative and class nonsingular for $A$, and $U \in \mathcal{M}$. Notice that if $L$ is a nonsingular M-matrix, then $L$ satisfies the given conditions, thus we are considering a larger set of LU factorizations than were considered in [13]. We would like $L$ and $U$ to be as close to lower and upper triangular respectively as possible. We begin by showing that certain access relationships must be present in $\mathcal{G}(U)$.

Theorem 4.1 (Minimality) Let $A \in \mathcal{M}$ and $U \in \mathcal{U}(A)$. Let $S_{1}, S_{2}, \ldots S_{m}$ be the singular classes of $A$. Then for each $i \in\langle m\rangle$ and $j \in\langle n\rangle$, if $j$ has access to $S_{i}$ in $\mathcal{G}(A)$, then $j$ has access to some vertex $q \in S_{i}$ in $\mathcal{G}(U)$.

Proof. Assume $j$ has access to $S_{i}$ in $\mathcal{G}(A)$. Since $A_{S_{i} S_{i}}$ is irreducible, $j$ has access in $\mathcal{G}(A)$ to every vertex of $S_{i}$. Let $Q_{i}$ be any final class of $U_{S_{i} S_{i}}$. For any $q \in Q_{i}$, we see that $j$ has access to $q$ in $\mathcal{G}(A)$. Choose $B \in \mathcal{N}_{A}$ such that $B A=U$. Applying [9, Theorem 3.7] we see that $j$ has access to $q$ in $\mathcal{G}(U)$.

Next we show that there is a $U \in \mathcal{U}(A)$, for which the only nonzeros below the diagonal of $U$ correspond to the required access relationships described in Theorem 4.1. To optimize on the placement of these zeros, for each $S_{i}$, we would like the vertex $q$ in Theorem 4.1 to be $\mu_{i}$. The desired $U$ can be found by performing Gaussian elimination until a zero diagonal element is encountered. When a zero diagonal element is encountered, that column should be skipped over, and the process continued. Thus we obtain a matrix $U$ which is upper triangular except for spurs below the diagonal corresponding to the columns $\mu_{i}$. We thus obtain a $U$ whose column envelope is small, where column envelope is defined similarly to the row envelope in $[4$, p. 708$]$.

Notice that using this algorithm the $L$ which is produced is a nonsingular lower triangular M-matrix.

Theorem 4.2 (Existence) Let $A \in \mathcal{M}$. Let $S_{1}, S_{2}, \ldots S_{m}$ be the singular classes of $A$, and let $\mu_{i}=\max \left(S_{i}\right)$. Set

$$
\chi=\left\{\left(j, \mu_{i}\right) \mid i \in\langle m\rangle, j>\mu_{i} \text { and } j \text { has access to } \mu_{i} \text { in } G(A)\right\} .
$$

Then $A$ admits a factorization $A=L U$, where $L \in \mathcal{M}$ is lower triangular and nonsingular, and $U \in \mathcal{M}$ is such that for all $j>k, u_{j k}=0$ whenever $(j, k) \notin \chi$, and $u_{j j}=0$ if and only if $j=\mu_{i}$ for some $i \in\langle m\rangle$. 
Proof. Let $A^{\varepsilon}$ be the matrix obtained from $A$ by adding $\varepsilon$ to the $\left(\mu_{i}, \mu_{i}\right)$ - th element in $A$, for each $i \in\langle m\rangle$. Then for any $\varepsilon>0$ and any $i \in\langle m\rangle, A_{S_{i} S_{i}}^{\varepsilon}$ is nonsingular by [1, Theorem 4.16(2) and Theorem 2.7]. Hence $A^{\varepsilon}$ is a nonsingular M-matrix and thus all the diagonal elements of $A^{\varepsilon}$ are positive. For each $i \notin\left\{\mu_{1}, \ldots, \mu_{m}\right\}$, and for each $j>i$, use multiplication by elementary matrices on the left to add appropriate multiples of row $i$ to row $j$ so as to reduce $A^{\varepsilon}$ to a matrix for which the $(j, i)-t h$ element is 0 . Since the matrix formed at each step of this process is also a nonsingular M-matrix, we will not encounter a nonzero diagonal element as we proceed in this fashion.

Notice that since row $\mu_{k}, k \in\langle m\rangle$, is not used as a pivot row, the elementary matrices will be independent of $\varepsilon$. By proceeding in this manner we can produce a matrix $U^{\varepsilon}$ such that $u_{j i}^{\varepsilon}=0$ whenever $i \notin\left\{\mu_{1}, \ldots, \mu_{m}\right\}$ and $j>i$. Moreover, $\mathcal{G}\left(U^{\varepsilon}\right) \subseteq \overline{\mathcal{G}(A)}$.

The off diagonal elements of $U^{\varepsilon}$ remain nonpositive, hence $U^{\varepsilon}$ is a Zmatrix. Since the leading principal minors of $A^{\varepsilon}$ are positive, and adding a multiple of one row to another does not change the determinant, all the leading principal minors of $U^{\varepsilon}$ are positive. Hence $U^{\varepsilon}$ is a nonsingular Mmatrix. Since $\varepsilon$ only appears on the diagonal of $U^{\varepsilon}, U=\lim _{\varepsilon \rightarrow 0} U^{\epsilon}$ is an M-matrix such that $u_{j k}=0$ whenever $(j, k) \notin \chi$. It is easy to see that if we take $L$ to be the inverse of the product of the elementary matrices used above (in the appropriate order) then $L$ is a nonsingular lower triangular M-matrix and $A=L U$. Since all of the diagonal elements of $A$ where used as pivots, except for the $\left(\mu_{i}, \mu_{i}\right)-t h$, they must be nonzero both in $A$ and in $U$. Since $A_{S_{i} S_{i}}$ is singular, it must be that $U_{S_{i} S_{i}}$ is singular. By the construction of $U, U_{S_{i} S_{i}}$ is an upper triangular matrix with nonzeros on the diagonal except possibly for $u_{\mu_{i} \mu_{i}}$, hence it must be that $u_{\mu_{i} \mu_{i}}=0$. Thus $U$ has the desired pattern.

Example 4.3 Let

$$
A=\left[\begin{array}{cccccccc}
1 & -1 & 0 & 0 & 0 & 0 & 0 & 0 \\
-1 & 1 & 0 & 0 & 0 & -1 & 0 & 0 \\
-1 & 0 & 2 & -2 & 0 & 0 & -1 & 0 \\
0 & -1 & -1 & 1 & 0 & 0 & -2 & -1 \\
0 & 0 & 0 & -1 & 1 & 0 & 0 & 0 \\
0 & 0 & 0 & 0 & 0 & 0 & 0 & 0 \\
0 & 0 & 0 & 0 & 0 & -1 & 1 & 0 \\
0 & 0 & 0 & 0 & 0 & -1 & -1 & 1
\end{array}\right]
$$


Then $\mu_{1}=2, \mu_{2}=4, \mu_{3}=6$ and $\chi=\{(3,2),(4,2),(5,2),(5,4),(7,6)$, $(8,6)\}$ Using our algorithm we get

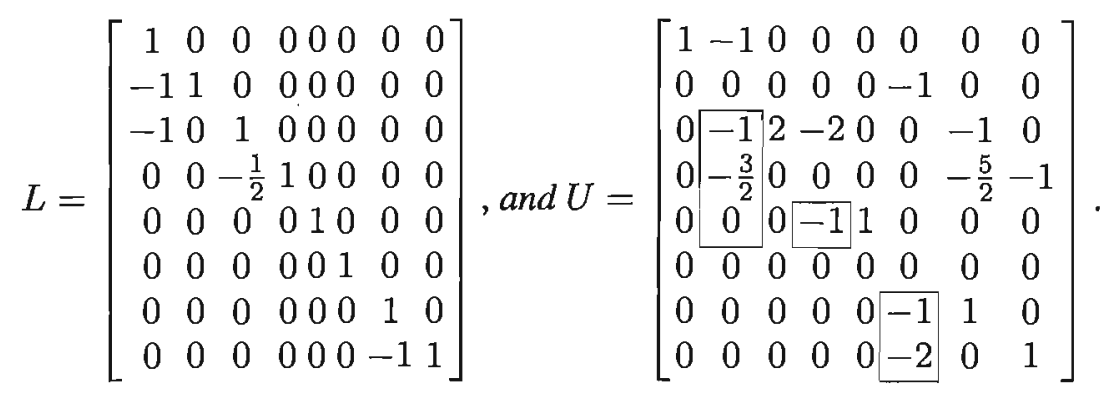

The boxes indicate the positions of potential nonzero elements below the diagonal of $U$. Notice that even though $(5,2) \in \chi, u_{5,2}=0$. Theorem 4.2 only asserts that the elements in $\chi$ are potentially nonzero.

Remark 4.4 Theorem 4.1 gives a lower bound on the number of nonzeros which must occur below the diagonal of $U$. Let $S_{1}, \ldots S_{m}$ be the singular classes of $A$. Let $\mu_{i}=\max \left(S_{i}\right), i \in\langle m\rangle$. Let

$$
R_{i}=\left\{j \in\langle n\rangle \mid j>\mu_{i} \text { and } j \text { has access to } \mu_{i} \text { in } \mathcal{G}(A)\right\} .
$$

Let

$$
R=\cup_{i=1}^{n} R_{i} .
$$

Then $|R| \leq$ the number of nonzeros below the diagonal of $U$. Moreover, Theorem 4.2 shows that there is a factorization of $A$ into a nonsingular lower triangular $\mathrm{M}$-matrix $L$ and a block upper triangular $\mathrm{M}$-matrix $U$, for which the number of nonzeros below the diagonal of $U \leq \sum_{i=1}^{m}\left|R_{i}\right|$. The next example shows that if the $R_{i}$ have nonempty pairwise intersection, then the number of nonzeros corresponding to this duplication is not identified by our theorems.

Example 4.5 Consider

$$
A=\left[\begin{array}{ccc}
0 & 0 & 0 \\
-1 & 0 & 0 \\
0 & -1 & 1
\end{array}\right], \quad B=\left[\begin{array}{ccc}
0 & 0 & 0 \\
-1 & 0 & 0 \\
-1 & -1 & 1
\end{array}\right]
$$

Both $A$ and $B$ have singular classes $S_{1}=\{1\}, S_{2}=\{2\}$, and in both $\mathcal{G}(A)$ and $\mathcal{G}(B)$, vertices 2 and 3 access $S_{1}$, and vertex 3 accesses $S_{2}$. Using the notation of Remark 4.4, $R_{1}=\{2,3\}, R_{2}=\{3\}$, and $R=\{2,3\}$. By Theorem 4.1, there must be at least 2 nonzero subdiagonal elements in $U$ for any block LU factorization of $A$ or $B$ (where $L$ and $U$ satisfy the conditions of Theorem 4.1), and by Theorem 4.2, there is an LU factorization of $A$ and an LU factorization of $B$, each with at most 3 subdiagonal elements in the $U$ 
and with the $L$ a nonsingular lower triangular M-matrix. Notice that if we factor $A=I A$, then $U=A$ has 2 nonzeros below the diagonal. However it can be shown that for any factorization of $B=L U$ (where $L$ and $U$ satisfy the conditions of Theorem 4.1), $U$ will have at least 3 nonzeros below the diagonal.

Our next theorem characterizes the M-matrices $A$ such that $A=L U$ where $L, U \in \mathcal{M}$ with $L$ nonsingular and lower triangular and $U$ upper triangular. The equivalence of parts (ii) and (iii) of this theorem appear in [13].

Theorem 4.6 Let $A \in \mathcal{M}$. Then the following are equivalent:

(i) There exists $U \in \mathcal{U}(A)$ such that $U$ is upper triangular.

(ii) A admits a factorization $A=L U$, where $L$ is a nonsingular lower triangular $M$-matrix and $U$ is an upper triangular $M$-matrix.

(iii) Let $m, T_{i}$, and $\mu_{i}$ be as in Definition 2.1. Then $T_{i}=\left\{\mu_{i}\right\}, \forall i \in\langle m\rangle$.

Proof. Follows from Theorem 4.1 and Theorem 4.2.

\section{Block LBU factorizations}

Using Theorem 4.2 we can factor $A=L B U$ into the product of a nonsingular lower triangular M-matrix $L$ with a block diagonal M-matrix $B$ and a nonsingular upper triangular $\mathrm{M}$-matrix $U$. Since $L$ and $U$ are nonsingular lower and upper triangular M-matrices, standard methods can be used to solve the parts of the system in which they are involved. In general, $B$ will be a sparse matrix since its only nonzero off-diagonal entries occur in rows and columns corresponding to the ends of singular classes. In this specialised case it may be possible to use sparse matrix techniques to solve the part of the system involving $B$.

Theorem 5.1 Let $A \in \mathcal{M}$. Let

$$
\begin{gathered}
\chi=\left\{(j, j) \mid j \in\langle n\rangle \backslash\left\{\mu_{i} \mid i \in\langle m\rangle\right\}\right\} \\
\cup\left\{\left(j, \mu_{i}\right) \mid i \in\langle m\rangle, j>\mu_{i} \text { and } j \text { has access to } \mu_{i} \text { in } \mathcal{G}(A)\right\} \\
\cup\left\{\left(\mu_{i}, j\right) \mid i \in\langle m\rangle, j>\mu_{i} \text { and } j \text { is accessed by } \mu_{i} \text { in } \mathcal{G}(A)\right\} .
\end{gathered}
$$

Then $A$ admits a factorization $A=L B U$ where $L$ is a nonsingular lower triangular $M$-matrix, $U$ is a nonsingular upper triangular $M$-matrix, and $B$ is an $M$-matrix such that $b_{j k}=0$ whenever $(j, k) \notin \chi$. Moreover, if $L$ and $U$ are nonsingular $M$-matrices, this is the finest block structure that $B$ can have (i.e it is impossible to subdivide the blocks of $B$ and obtain a block diagonal matrix). 
Proof. Use Theorem 4.2 to factor $A=L V$ where $L$ is a nonsingular Mmatrix and $V$ is an M-matrix such that for all $j>k, v_{j k}=0$ whenever $(j, k) \notin\left\{\left(j, \mu_{i}\right) \mid i \in\langle m\rangle, j>\mu_{i}\right.$ and $j$ has access to $\mu_{i}$ in $\left.\mathcal{G}(A)\right\}$. Notice that the singular classes of $V$ are just $\mu_{1}, \ldots, \mu_{m}$, and $\mathcal{G}(V) \subseteq \overline{\mathcal{G}(A)}$. Using the algorithm outlined in the proof of Theorem 4.2, we can factor $V^{\mathrm{T}}=X Y$ where $X$ is an nonsingular lower triangular $\mathrm{M}$-matrix, and $Y$ is an $\mathrm{M}$-matrix such that for all $j>k, y_{j k}=0$ whenever $(j, k) \notin\left\{\left(j, \mu_{i}\right)|i \in\langle m\rangle, j\rangle\right.$ $\mu_{i}$ and $j$ has access to $\mu_{i}$ in $\left.\mathcal{G}(A)\right\}$. Since rows $\mu_{1}, \ldots, \mu_{m}$ are not used as pivot rows, and all other rows of $V^{\mathrm{T}}$ have zeros to the right of the diagonal, $Y$ will also satisfy the property that for all $j>k, y_{k j}=0$ whenever $(j, k) \notin\left\{\left(j, \mu_{i}\right) \mid i \in\langle m\rangle, j>\mu_{i}\right.$ and $j$ has access to $\mu_{i}$ in $\left.\mathcal{G}(A)\right\}$. Let $B=Y^{\mathrm{T}}$ and $U=X^{\mathrm{T}}$. Then $A=L B U$ is as claimed. By Theorem 4.1, the block structure exhibited by $B$ is the finest possible with $L$ and $U$ nonsingular $\mathrm{M}$-matrices.

Example 5.2 Let

$$
A=\left[\begin{array}{cccccccc}
1 & -1 & 0 & 0 & 0 & 0 & 0 & 0 \\
0 & 0 & -1 & 0 & 0 & 0 & 0 & 0 \\
0 & 0 & 1 & 0 & -1 & 0 & 0 & 0 \\
0 & 0 & 0 & 1 & 0 & 0 & 0 & 0 \\
0 & 0 & -1 & 0 & 1 & -1 & 0 & 0 \\
0 & 0 & 0 & 0 & 0 & 0 & 0 & 0 \\
0 & 0 & 0 & 0 & 0 & 0 & 0 & -1 \\
-1 & 0 & 0 & 0 & 0 & 0 & 0 & 0
\end{array}\right]
$$

as in Example 3.13 Then

$$
\mu_{1}=2, \mu_{2}=5, \mu_{3}=6, \mu_{4}=7, \mu_{5}=8,
$$

and

$$
\begin{aligned}
\chi= & \{(1,1),(3,3),(4,4),(2,3),(2,4),(2,5),(2,6),(7,2),(8,2),(5,6), \\
& (7,5),(8,5)(7,6),(8,6),(7,8)\} .
\end{aligned}
$$

Using the algorithm outlined above Theorem 4.2 we get $A=L V$. Then using the algorithm to factor $V^{\mathrm{T}}=U^{\mathrm{T}} B^{\mathrm{T}}$ we get

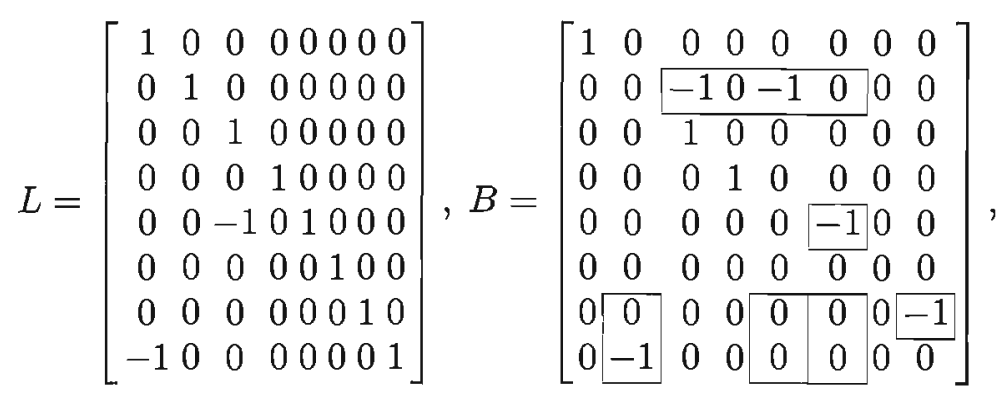




$$
U=\left[\begin{array}{cccccccc}
1 & -1 & 0 & 0 & 0 & 0 & 0 & 0 \\
0 & 1 & 0 & 0 & 0 & 0 & 0 & 0 \\
0 & 0 & 1 & 0 & -1 & 0 & 0 & 0 \\
0 & 0 & 0 & 1 & 0 & 0 & 0 & 0 \\
0 & 0 & 0 & 0 & 1 & 0 & 0 & 0 \\
0 & 0 & 0 & 0 & 0 & 1 & 0 & 0 \\
0 & 0 & 0 & 0 & 0 & 0 & 1 & 0 \\
0 & 0 & 0 & 0 & 0 & 0 & 0 & 1
\end{array}\right]
$$

The boxes in $B$ indicate the only possible positions for nonzero off diagonal entries.

Acknowledgements. We would like to thank Michael Tsatsomeros and an anonymous referee for helpful comments and suggestions.

\section{References}

1. Berman, A., Plemmons, R.J. (1979): Nonnegative Matrices in the Mathematical Sciences. Academic Press, New York

2. Birkhoff, G. (1967): Lattice Theory, American Mathematical Society Colloquium Publications, Volume XXV, Third edition

3. Fiedler, M., Ptak, V. (1962): On matrices with nonpositive off-diagonal elements and positive principal minors. Czech. Math. J., 12:382-400

4. George, A., Pothen, A. (1977): An analysis of spectral envelope reduction via quadratic assignment problems. SIAM J. Matrix Analysis and Applications, 18:706-732

5. Grätzer, G. (1978): General Lattice Theory. Academic Press

6. Higham, N.J. (1996): Accuracy and Stability of Numerical Algorithms. SLAM

7. Hom, R.A., Johnson, C.R. (1985): Matrix Analysis. Cambridge University Press

8. Kuo, I. (1977): A note on factorizations of singular M-matrices. Linear Algebra and its Applications, $16: 217-220$

9. McDonald, J.J. (1994): A product index theorem with applications to splittings of M-matrices. Linear Algebra and its Applications, 197/198:511-530

10. Rothblum, U.G. (1975): Algebraic Eigenspaces of Nonnegative Matrices. Linear Algebra its Applications, 12:281-292

11. Schneider, H. (1984): Theorems on $M$-splittings of a Singular M-matrix Which Depend on Graph Structure. Linear Algebra and its Applications, 58:407-424

12. Schneider, H. (1986): The Influence of the Marked Reduced Graph of a Nonnegative Matrix on the Jordan Form and on related Properties: A Survey. Linear Algebra and its Applications, 84:161-189

13. Varga, R.S., Cai, D.-Y. (1981): On the LU Factorization of M-matrices. Numer. Math., 38:179-192 